\title{
The spin temperature of warm interstellar H I
}

\author{
H. Liszt ${ }^{1}$ \\ National Radio Astronomy Observatory, 520 Edgemont Road, Charlottesville, VA 22903-2475, USA \\ Received 1 February 2001 / Accepted 15 March 2001

\begin{abstract}
Collisional excitation of the $\lambda 21 \mathrm{~cm}$ hyperfine transition is not strong enough to thermalize it in warm neutral ("intercloud") interstellar gas, which we show by simultaneously solving the equations of ionization and collisional equilibrium under typical conditions. Coupling of the $\lambda 21 \mathrm{~cm}$ excitation temperature and local gas motions may be established by the Ly- $\alpha$ radiation field, but only if strong Galactic Ly- $\alpha$ radiation permeates the gas in question. The Ly- $\alpha$ radiation tends to impart to the gas its own characteristic temperature, which is determined by the range of gas motions that occur on the spatial scale of the Ly- $\alpha$ scattering. In general, the calculation of $\mathrm{H}$ I spin temperatures is a more difficult and interesting problem than might have been expected,
\end{abstract} \\ as is any interpretation of $\mathrm{H} \mathrm{I}$ spin temperature measurements.
}

Key words. interstellar medium: general

\section{Introduction}

It is an article of faith among $\mathrm{H}$ I observers that the spin (excitation) temperature $T_{\mathrm{sp}}$ of the ground-state $\lambda 21 \mathrm{~cm}$ hyperfine transition is equal to the kinetic temperature of the ambient gas. This belief of course arises from the weakness of the transition, the consequent long lifetime against spontaneous emission $\left(A_{21}=2.8510^{-15} \mathrm{~s}^{-1}\right.$ for $\mathrm{H} \mathrm{I}$ ), and the easily-demonstrated dominance of particle collisions with other $\mathrm{H}$-atoms in cool gas. Much of our basic understanding of the phases of the ISM derives directly from measurements of the $\mathrm{H}$ I spin temperature, for instance by comparing nearby or overlapping absorption and emission profiles (Dickey et al. 1978; Payne et al. 1982).

But spin temperature measurements often seem to imply unphysical kinetic temperatures: not wildly so, say negative or infinite, but definitely in a range - 1000 to $5000 \mathrm{~K}$ - where the interstellar gas is unstable in multiphase models (Wolfire et al. 1995; McKee \& Ostriker 1977) and therefore should be so short-lived as to be unobservable. One might be tempted to disparage either theory or the data but Davies \& Cummings (1975) took a somewhat less doctrinaire view and showed that, for some assumed combinations of conditions chosen to be representative of gas which might produce pulsar dispersion measures, the density of collision partners in warm or intercloud gas is simply too small to thermalize the line. Although little attention seems to have been paid to this warning, it suggests that we remain open to the possibility that blind faith in the equality of the spin and kinetic temperatures might be misplaced.

Send offprint requests to: H. S. Liszt, e-mail: hliszt@nrao.edu
Calculation of the $\mathrm{H}$ I spin temperature turns out to be a remarkably complex problem, involving intimate knowledge of the ionization and phase structure of the ISM, as well as its topology. Here we update and expand upon the discussion of Davies \& Cummings (1975) by actually calculating the ionization and collisional excitation in interstellar $\mathrm{H}$ I regions, in the process demonstrating the inability of particle collisions to thermalize the $\lambda 21 \mathrm{~cm}$ transition. But we also consider an important mechanism which Davies \& Cummings (1975) ignored, whereby the Ly- $\alpha$ radiation field threading and partly produced by the gas tends to impart to the $\lambda 21 \mathrm{~cm}$ transition its own effective temperature. Ly- $\alpha$ photons acquire this temperature while undergoing large numbers $\left(10^{7}\right.$ or more) of repeated scatterings on many spatial scales ranging upward from the line-center mean free path, $0.11\left(T_{\mathrm{D}} / 100 \mathrm{~K}\right)^{0.5} / n_{\mathrm{H}} \mathrm{AU}$ ( $T_{\mathrm{D}}$ is the Doppler temperature), and it is therefore representative of the motions (thermal, turbulent, etc.) which are established in the gas on those scales.

That excitation by light dominates hyperfine excitation locally around individual stars is well known in the context of scattering of Solar Ly- $\alpha$ radiation: Braskén \& Kyrölä (1998), for instance, point out that Solar Ly- $\alpha$ radiation dominates in local intercloud H I within $1000 \mathrm{AU}$ of the Sun, and that the sphere of influence of an O-star would necessarily be much larger. In the interstellar context, we will refer to excitation of the hyperfine line by scattered Ly- $\alpha$ radiation as the Wouthuysen-Field mechanism or WF effect following the discussions in Wouthuysen (1952) and Field $(1958,1959)$ and we find that it opens up interesting possibilities for the spin temperature. At low density in the intercloud medium, warm neutral H I may (or may not) be Dopplerized by Galactic Ly- $\alpha$ photons but 
is certainly not thermalized by local particle collisions. In general, the importance which is assigned to $\mathrm{Ly}-\alpha$ excitation in intercloud gas depends directly on the topology and large-scale structure which is assumed to apply in the interstellar medium.

The plan of the current discussion is as follows. In Sect. 2 we show what is needed to calculate the excitation of the hyperfine transition. In Sect. 3 we lay out the basics of the ionization equilibrium calculations which are needed to determine the densities of the main collision partners (electrons and neutral $\mathrm{H}$-atoms) under given conditions of local gas density and gas thermal pressure. These calculations also serve to determine the locally-produced field of Ly- $\alpha$ photons. In Sect. 4 we discuss results of the combined ionization and particle excitation calculations. In Sect. 5 we introduce the behaviour of the internally-generated Ly- $\alpha$ radiation field, and conclude that it also is not strong enough to influence the hyperfine excitation in neutral gas. In Sect. 6 we discuss the effect of the much stronger galactic Ly- $\alpha$ photon field on the $\mathrm{H} \mathrm{I}$ in various multiphase models of the ISM.

\section{Hyperfine excitation}

Discussions of the excitation of the $\lambda 21 \mathrm{~cm}$ transition generally follow that of Field (1958) who distinguished among three types of excitation; radiative, by a radiation field around the rest wavelength of the hyperfine transition having a characteristic brightness temperature $T_{\mathrm{R}}$ as seen in the gas $\left(T_{\mathrm{R}}=2.73 \mathrm{~K}\right.$ here $)$; collisional with a total downward rate $R_{21}^{\mathrm{c}}\left(\mathrm{s}^{-1}\right)$ at kinetic temperature $T_{\mathrm{K}}$; and radiative in the Ly- $\alpha$ photon field, with a net downward rate $R_{21}^{\alpha}$, at an effective temperature $T_{\mathrm{L}}$. Field derived an expression for the Ly- $\alpha$ excitation which was used in an equivalent form by Bahcall \& Ekers (1969) and which will be discussed below.

In these terms the spin (i.e. excitation) temperature of the $\lambda 21 \mathrm{~cm}$ line can be expressed as

$T_{\mathrm{sp}}=\left(T_{\mathrm{R}}+y_{\mathrm{c}}+y_{\alpha}\right) /\left(1+y_{\mathrm{c}} / T_{\mathrm{K}}+y_{\alpha} / T_{\mathrm{L}}\right)$

with

$y_{\mathrm{c}}=T_{21} R_{21}^{\mathrm{c}} / A_{21}$,

$y_{\alpha}=T_{21} R_{21}^{\alpha} / A_{21}$

and where $T_{21} \equiv h \nu_{21} / k=0.068 \mathrm{~K}, A_{21}=$ $2.8510^{-15} \mathrm{~s}^{-1}$.

Equation (1) shows that $R_{21}>15 T_{\mathrm{K}} A_{21}$ is required in order to thermalize the transition.

\subsection{Collisional excitation by electrons, protons, and neutral $\mathrm{H}$-atoms}

The required rate $R_{21}^{\mathrm{c}}$ is the sum of the downward collision rates over all collision partners (H-atoms, protons, and electrons). The downward rate constant for interactions with electrons is taken from Smith (1966): we note that it is well-represented by the functional form

$\log \gamma_{21}^{\mathrm{e}}\left(T_{\mathrm{K}}\right)=-9.607+\log \left(\sqrt{T_{\mathrm{K}}}\right) \exp \left(-\left(\log T_{\mathrm{K}}\right)^{4.5} / 1800\right)$

for $T_{\mathrm{K}} \leq 10^{4} \mathrm{~K}$ and

$\gamma_{21}^{\mathrm{e}}\left(T_{\mathrm{K}} \geq 10^{4} \mathrm{~K}\right)=\gamma_{21}^{\mathrm{e}}\left(10^{4} \mathrm{~K}\right)$

over the range considered here (the logs are base 10 and the units of $\gamma_{21}^{\mathrm{e}}$ are $\left.\mathrm{cm}^{3} \mathrm{~s}^{-1}\right)$.

Smith (1966) also gives the rate coefficient for proton de-excitation, which is just 3.2 times larger than that for neutral atoms at $T_{\mathrm{K}}>30 \mathrm{~K}$. We used $\gamma_{21}^{\mathrm{p}}=3.2 \gamma_{21}^{\mathrm{H}}$ but employed the more recent neutral atom de-excitation rates of Allison \& Dalgarno (1969) which we fit in piece-wise continuous fashion, following their tabular presentation. Excitation by protons is unimportant because it is so much weaker than that by electrons at the same temperature and because $n_{\mathrm{e}}>n_{\mathrm{p}}$ under all conditions.

The results of Allison \& Dalgarno (1969) are given up to $1000 \mathrm{~K}$ at which point they are increasing as $T_{\mathrm{K}}{ }^{0.33}$, but with a negative second derivative. The neutral-atom rate coefficients of Smith (1966) increase somewhat faster for $T_{\mathrm{K}}>1000 \mathrm{~K}$ with a slightly positive second derivative, but are not substantially different in the mean. We extrapolated the rate coefficients of Allison \& Dalgarno (1969) to higher temperature as $T_{\mathrm{K}}{ }^{0.33}$. In this regime the excitation is increasingly dominated by electrons and the results reported here are not noticeably influenced by the manner of the extrapolation.

\subsection{Excitation by the $L y-\alpha$ photon field}

Recoil - momentum conservation - at each scattering causes the field of Ly- $\alpha$ photons to have a slope (color temperature) at the line center which corresponds to the Doppler temperature of the ambient medium (Field 1959; Adams 1971a); Ly- $\alpha$ photons sample the gas kinematics and then tend to impart to the hyperfine transition a corresponding excitation temperature. Bahcall \& Ekers (1969) and Urbaniak \& Wolfe (1981) employed this mechanism to show how the $\mathrm{H}$ I spin temperature could remain low, rendering $\lambda 21 \mathrm{~cm}$ absorption detectable, even in neutral gas near quasars. In cases where the gas kinematics are affected by non-thermal motion, this will be reflected in the spin temperature, depending on the relative importance of collisional and Ly- $\alpha$ excitation.

Field (1958) considered detailed balance in the various transitions between sublevels which constitute the Ly- $\alpha$ line and arrived at the following expression for $y_{\alpha}$

$y_{\alpha}=\left(c T_{21} / 36 \pi\right)\left(m_{\mathrm{H}} / k T_{\mathrm{D}}\right)^{0.5} \lambda_{\alpha}^{3}\left(A_{\alpha} / A_{21}\right) n_{\alpha}$

where $A_{\alpha}$ is the spontaneous emission rate $\left(4.710^{8} \mathrm{~s}^{-1}\right.$ corresponds to the mean Ly- $\alpha$ absorption $f$-value $f=$ $0.4167), n_{\alpha}$ is the number density of Ly- $\alpha$ photons at the line center and $T_{\mathrm{D}}$, the Doppler temperature, represents the width of the Gaussian core of the line. $T_{\mathrm{D}}$ is defined in the usual way (Leung \& Liszt 1976) by the expression

$2 k T_{\mathrm{D}} / m_{\mathrm{H}}=2 k T_{\mathrm{K}} / m_{\mathrm{H}}+v_{\text {turb }}{ }^{2}$ 
leading to $T_{\mathrm{D}}=T_{\mathrm{K}}+121 \mathrm{~K}\left(v_{\text {turb }} / 1 \mathrm{kms}^{-1}\right)^{2}$. This expression accords with the definition used by Payne et al. (1982) $T_{\mathrm{D}}=21.86 \mathrm{~K} \mathrm{~W}^{2}$ where $W$ is the $F W H M\left(\mathrm{~km} \mathrm{~s}^{-1}\right)$ and differs from the earlier usage of Dickey et al. (1978), $T_{\mathrm{D}}=121 \mathrm{~K} W^{2}$ taking $W$ as the $H W H M$. The Doppler temperatures shown by Dickey et al. (1978) are actually too large by a factor $2_{*} \ln (2) \approx 1.4$.

Evaluating the constants in Eq. (2) we find

$y_{\alpha}=5.9010^{11} n_{\alpha} T_{\mathrm{D}}^{-0.5}$.

\section{Ionization equilibrium in $\mathrm{H} I$ regions}

\subsection{Calculations}

To obtain number densities of the various collision partners which determine the equilibrium of the hyperfine transition it is necessary to calculate the ionization equilibrium of a dilute gas. We duplicated the relevant portions of the work of Wolfire et al. (1995) which is a very comprehensive study of two-phase equilibrium in the interstellar medium and must be regarded as the standard reference on this subject. That is, we consider a gas consisting of hydrogen, helium and carbon, ionized by soft X-rays and cosmic rays (and their secondary electrons) which recombines in both the gas phase and on small grains (following the formalism of Draine \& Sutin 1987). This calculation is fully described in the original reference and a detailed discussion will not be repeated here. We note that it uses Solar metallicity, no appreciable gas-phase depletion of carbon $\left([\mathrm{C}] /[\mathrm{H}]=3.310^{-4}\right)$, and a primary cosmic ray ionization rate $\zeta_{\mathrm{H}}^{\mathrm{cr}}=1.710^{-17} \mathrm{~s}^{-1}$. The ionization fractions are overestimates by factors of $2-3$ in higher-density gas if gas-phase depletion is important.

Wolfire et al. (1995) parametrized their model in terms of a column density of hydrogen nuclei $N w$ which attenuates part of the soft X-ray flux; their standard model takes a value $N w=10^{19} \mathrm{~cm}^{-2}$ for this quantity, with factor of ten variations in either sense as subsidiary examples. To model the X-ray ionization rate, we calculated the total attenuation at each energy over a gas column $N w$ (due to all the important elements, not just those whose ionization is calculated) using the absorption cross-sections of Balucinska-Church \& McCammon (1992) and modulated the free-space X-ray spectrum accordingly. The product of this attenuated and modulated X-ray spectrum and the absorption cross section is then integrated over energy to give the primary ionization rate for hydrogen and helium. We also calculated the mean photoelectron energy for use in determining the secondary ionization; this is typically 40-50 eV as for cosmic-ray events.

\subsection{Recombination coefficients}

We took recombination coefficients for various species from the 1999 update of the UMIST reaction database. They are expressed in the form $\alpha_{Y}=a_{Y} \times\left(T_{\mathrm{K}} / 300\right)^{-b_{Y}}$ where the coefficients are given in Table 1. Hydrogen of
Table 1. Recombination rate coefficients

\begin{tabular}{lcc}
\hline Species & $a\left(\mathrm{~cm}^{3} \mathrm{~s}^{-1}\right)$ & $b$ \\
\hline $\mathrm{H}_{n>1}$ & $3.5010^{-12}$ & 0.75 \\
$\mathrm{H}_{2} \mathrm{~S}$ & $1.5410^{-13}$ & $0.54^{a}$ \\
$\mathrm{He}$ & $2.3610^{-12}$ & 0.64 \\
$\mathrm{C}$ & $4.6710^{-12}$ & 0.60 \\
\hline
\end{tabular}

${ }^{a}$ Rate into the ${ }^{2} \mathrm{~S}$ level only, see Sect. 3.2.

course is a special case whereby the needed recombination coefficient is that into the $n=2$ state and higher, since recombinations into $n=1$ produce ionizing photons which are immediately reabsorbed in the surrounding gas. A special case is considered below whereby we require the recombination rate for production of Ly- $\alpha$ radiation. In this case, recombinations into the metastable $2 \mathrm{~S}$ state must be ignored, and the effective recombination rate is diminished by an amount which is given in the second entry of the table, taken from Martin (1988).

\subsection{Photoionization of carbon}

For this we take from the UMIST database the free space value $\zeta_{\mathrm{C}}^{\gamma}(0)=3.010^{-10} \mathrm{~s}^{-1}$. This can then be traced into a gas column as $\zeta_{\mathrm{C}}^{\gamma}\left(A_{V}\right)=\zeta_{\mathrm{C}}^{\gamma}(0) \exp \left(-2.42 A_{V}\right)$ following Black \& Dalgarno (1977) but we will consider only the case $A_{V}=0$. The major contribution to the ionization fraction for the warm gas considered here is generally from $\mathrm{H}$ and $\mathrm{He}$, not from carbon.

\subsection{Collisional ionization of hydrogen by electrons}

We used the electron-impact collisional ionization rates of Scholz \& Walters (1991). The reader is cautioned to ignore an unfortunate 10 order of magnitude error in labelling of the vertical scale of their Fig. 1, lest this crucial process be ignored.

\section{Ionization equilibrium and hyperfine excitation in the interstellar hydrogen}

\subsection{The ionization fraction as a function of gas pressure and density}

In Fig. 1 we show the results of our ionization calculations for four values of the gas thermal pressure, using the standard value $N w=10^{19} \mathrm{~cm}^{-2}$. The ionization fraction is shown as solid lines and the electron density is shown shaded. The kinetic temperature is traced across the set of four curves of varying pressure. Clearly, the ionization fraction in neutral gas is controlled by the total density, while the electron density - which varies remarkably little with $n_{\mathrm{H}}$ until the gas ionizes fully - is influenced somewhat by the pressure. The soft X-ray ionization rate scales the overall behaviour, albeit somewhat weakly (not shown).

The filled dark squares in Fig. 1 represent the limiting cases at high and low pressure for the model of two-phase 


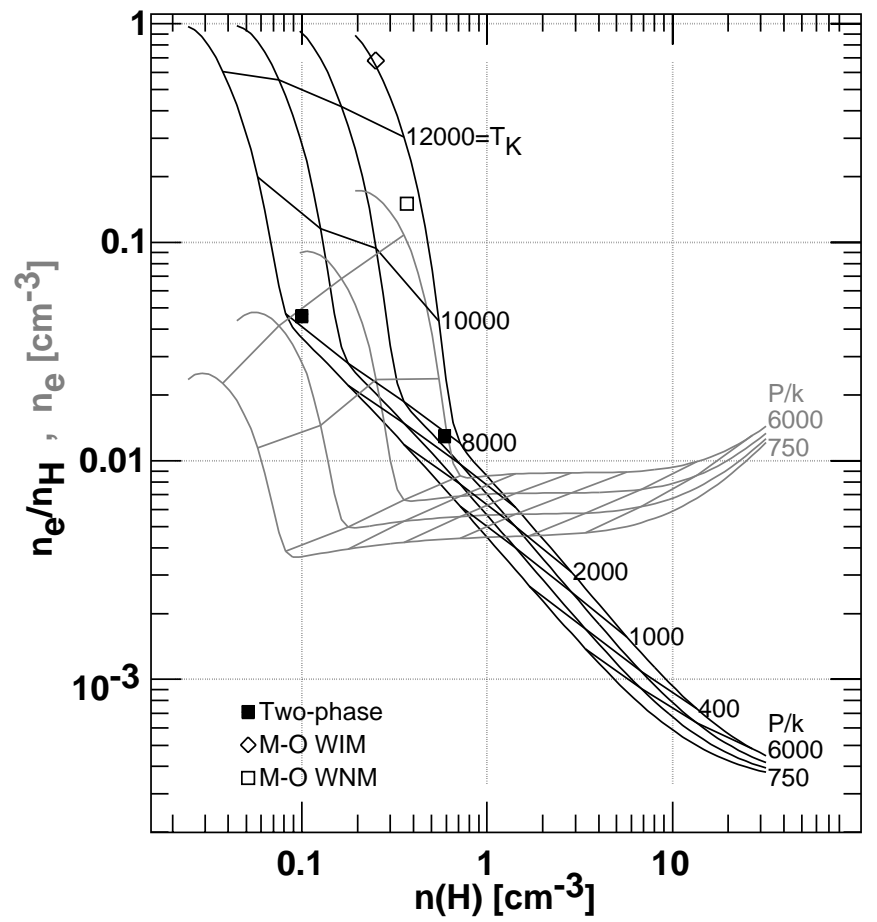

Fig. 1. Ionization equilibrium calculations in atomic gas. The full curves are plots of the ionization fraction $n_{\mathrm{e}} / n_{\mathrm{H}}$ where $n_{\mathrm{H}}$ is the number density of H-nuclei in all forms. Results are shown for four values of the total gas pressure $P / k=$ $750,1500,3000,6000 \mathrm{~cm}^{-3} \mathrm{~K}$ and the kinetic temperature is traced across each set of curves. The shaded curves are plots of the electron density. Open symbols represent the ionization fraction in warm neutral and warm ionized gas in the threephase model of McKee \& Ostriker (1977). The filled rectangles show the ionization fraction of warm neutral gas in the twophase calculations of Wolfire et al. (1995) for their standard case $N w=10^{19} \mathrm{~cm}^{-2}$

equilibrium from Wolfire et al. (1995). Table 2 gives the range of pressure and kinetic temperature over which two phases can coexist for various values of $N w$ and it shows that two-phase equilibrium is possible over a wider range in $T_{\mathrm{K}}$ than is sometimes considered when interpreting spin temperature measurements. Comparison of the table entries for the standard case $N w=110^{19} \mathrm{~cm}^{-2}$ with the locations of the filled dark symbols in the figure shows that our results mimic those of Wolfire et al. (1995), as intended. Both sets of calculations produce the same ionization at any given density and temperature.

In Fig. 1, two unfilled symbols represent the results of McKee \& Ostriker (1977) for the warm neutral and warm ionized gas (both at $8000 \mathrm{~K}$ ). The pressure in the McKeeOstriker model is $P / k=3700 \mathrm{~K} \mathrm{~cm}^{-3}$ but the ionization fraction in three-phase warm neutral and ionized gas is considerably higher than that in our calculations. McKee \& Ostriker (1977) explain in the caption to their Fig. 1 that the warm neutral medium can only be produced in appreciable quantity at the assumed pressure by assuming a higher than average value of the soft X-ray flux. In the context of our modelling, the soft X-ray flux would have to be increased by a factor of about 50 in order to give the

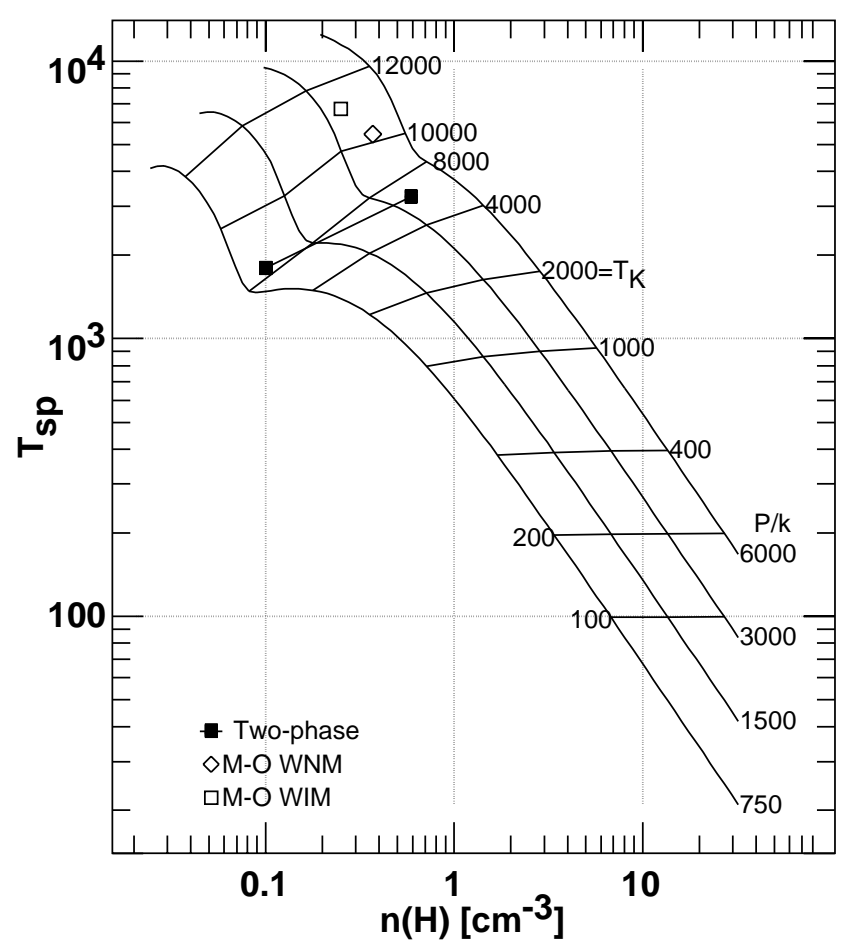

Fig. 2. The spin temperature of the H I $\lambda 21 \mathrm{~cm}$ transition resulting from the same conditions whose ionization equilibrium is shown in Fig. 1. Symbols represent results for warm gas in the two and three phase calculations of Wolfire et al. (1995) and McKee \& Ostriker (1977), see Fig. 1

Table 2. Two-phase spin and kinetic temperatures

\begin{tabular}{lccc}
\hline Model & $P / k\left(\mathrm{~cm}^{3} \mathrm{~K}\right)$ & $T_{\mathrm{K}}(\mathrm{K})$ & $T_{\mathrm{sp}}(\mathrm{K})$ \\
\hline$N w=10^{18} \mathrm{~cm}^{-2}$ & $1600-9990$ & $9200-5600$ & $3525-4620$ \\
$N w=10^{19} \mathrm{~cm}^{-2}$ & $990-3600$ & $8700-5500$ & $1800-3260$ \\
$N w=10^{20} \mathrm{~cm}^{-2}$ & $610-1500$ & $8200-4900$ & $1035-2020$ \\
Three-Phase & 3700 & 8000 & 5400 \\
\hline
\end{tabular}

same total ionization which occurs in warm, neutral gas in the McKee-Ostriker model. It is unfortunate that the basic parameters of the three-phase model have not been revised in so long.

\subsection{Evaluation of $T_{\mathrm{sp}}$}

Figure 2 shows that for kinetic temperatures above $2000 \mathrm{~K}$, and especially at $8000 \mathrm{~K}$, the particle excitation rate is too small to thermalize the $\lambda 21 \mathrm{~cm}$ line in any model of the ISM, including the McKee-Ostriker threephase picture. The extreme conditions assumed for the three-phase model result in $T_{\mathrm{sp}} \approx 5400 \mathrm{~K}$ in warm neutral gas with $T_{\mathrm{K}}=8000 \mathrm{~K}$. In the two-phase model, the spin temperature of warm gas would be smaller, 1800-3200 K for the standard conditions where $T_{\mathrm{K}}=8700-5500 \mathrm{~K}$ (the models at higher $T_{\mathrm{K}}$ have lower pressure and lower $\left.T_{\mathrm{sp}}\right)$. Table 2 shows the range of spin temperature which results from various conditions of two- and three-phase equilibrium. Figure 3 displays the $y$-factor for collisional 
excitation corresponding to the conditions shown in Figs. 1 and 2 . In the warm gas, $y_{\mathrm{c}} / T_{\mathrm{K}} \approx 1$, while $y_{\mathrm{c}} / T_{\mathrm{K}}>>1$ is the relevant condition for thermalization.

Physically, the subthermal excitation comes about because the condition for thermalization becomes ever more stringent at higher kinetic temperature, while the neutral particle excitation rate coefficient only increases as $T_{\mathrm{K}}{ }^{0.3}$ (Allison \& Dalgarno 1969). The neutral particle density declines as $1 / T_{\mathrm{K}}$ at constant pressure until the gas begins to ionize, at which point new particles enter the gas and the decline is even more rapid. Once the gas ionizes even slightly (a few percent), excitation by electrons begins to dominate and the remaining neutral atoms will be more strongly excited. But the transition from neutral to ionized gas (which increasingly precludes observation of the $\lambda 21 \mathrm{~cm}$ line) occurs at too low a density to preserve the thermalization of the line even when a very large soft $\mathrm{X}$-ray flux is asserted, as in the model of McKee \& Ostriker $(1977)^{1}$.

We conclude that in the context of these calculations, one simply cannot expect to observe spin temperatures which are directly indicative of the $8000 \mathrm{~K}$ kinetic temperature typically assumed (by observers) for warm gas. Instead, seemingly unphysical, intermediate values will appear, even when the contribution of warm gas can be isolated. This situation comes about mostly because the excitation is sub-thermal, but also because multi-phase equilbrium is not entirely oblivious of local conditions; the kinetic temperature of warm neutral gas can vary between 5000 and $9000 \mathrm{~K}$ in two-phase models depending on the pressure, elemental abundances, etc. Moreover, Wolfire et al. (1995) note that the time to reach thermal equilibrium in warm gas is too long to ensure that a steady-state actually occurs; rather, two-phase equilibrium is an ideal, toward which the ISM may tend. If seemingly forbidden spin temperatures $1000-5000 \mathrm{~K}$ are actually measured in the $\lambda 21 \mathrm{~cm}$ line, it is unfair and improper to impugn the multi-phase models on this account, because they predict them.

Finally, we note that the need to consider a wide range of spin temperatures in warm gas makes it harder to discern $T_{\mathrm{sp}}$ in cool gas when both are seen blended along the line of sight. Of course, lines of sight in which the contribution of cool gas is not strongly blended are almost unknown. The presence of lower $T_{\mathrm{sp}}$ in the intercloud medium would result in lower $T_{\mathrm{sp}}$ in line blends, perhaps producing another range of seemingly unphysical $T_{\mathrm{sp}}$ between $\approx 1000 \mathrm{~K}$ and the range expected for cool gas alone, 40-200 K (Wolfire et al. 1995).

\section{Excitation by the Ly- $\alpha$ photon field}

In the previous section we showed that the $\lambda 21 \mathrm{~cm}$ hyperfine line will not be thermalized by particle collisions in warm weakly ionized gas. But there is another means of

\footnotetext{
1 Perhaps this model might also be referred to as the Lake Woebegon solution, since it seems to assume that the soft xray field is everywhere brighter than average.
}

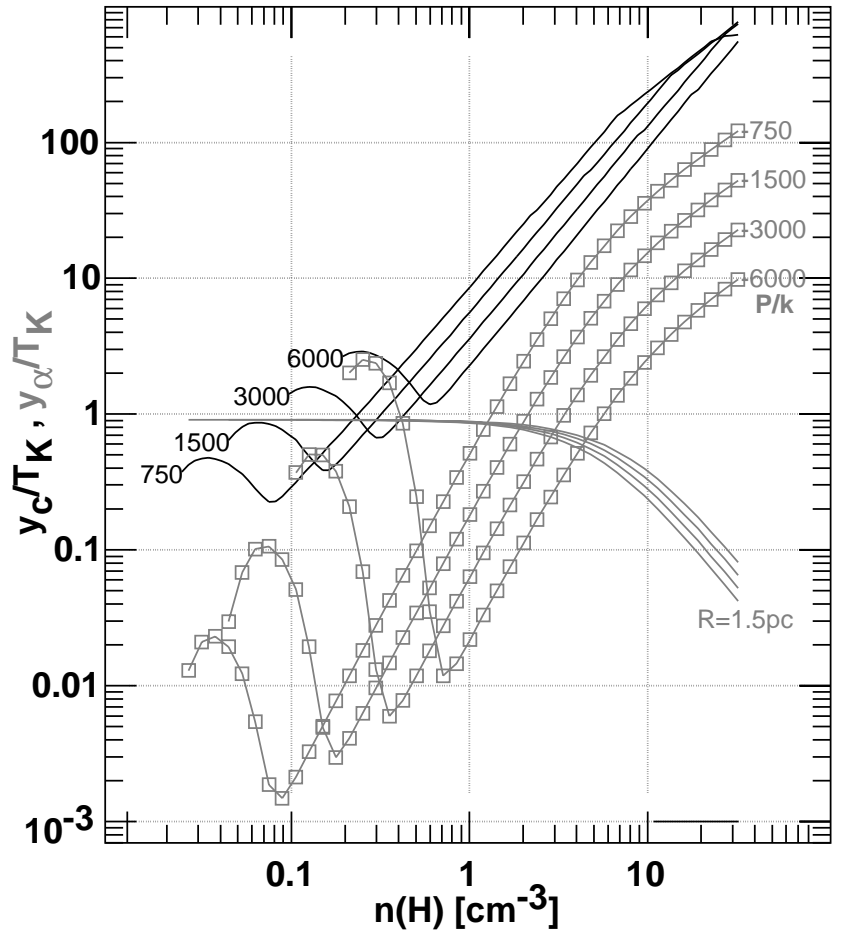

Fig. 3. The $y$-parameter for collisional (solid curves) and Ly- $\alpha$ excitation (shaded and chained curves) for a medium of assumed size $2 R=3 \mathrm{pc}$. The same 4 thermal pressures are employed here as in Figs. 1 and 2. The shaded curves marked by " $R=1.5 \mathrm{pc}$ " represent the correction factors, accounting for radiative transfer in lossy media, which have been applied to the Ly- $\alpha$ excitation following the discussion in Sect. 5.2; see also the following figure. The horizontal line at lower right shows where $N_{\mathrm{H}}=2 n_{\mathrm{H}} R=1-310^{20} \mathrm{~cm}^{-2}$

excitation available, the Wouthuysen-Field effect, which we now discuss.

\subsection{Ly- $\alpha$ scattering}

Our basic understanding of the behaviour of Ly- $\alpha$ photons in very optically thick media dates from the discussion of Field (1959) on relaxation in Doppler broadening and the numerical work of Adams (1972) on scattering including strong damping. The latter showed that the mean number of scatterings $\langle N\rangle$ of a photon before escape from a lossless medium never exhibits the behaviour expected of a pure one-dimensional random walk. Taking $\tau_{0}$ as the linecenter optical depth at the midplane of a semi-infinite slab viewed along the outward normal, Adams found instead that, at sufficiently high optical depth (see Hummer \& Kunasz 1980), $\langle N\rangle \approx \tau_{0}$. This seems to have been unexpected, even though identical behaviour is discussed by Field (1959), citing Zanstra (1949), following essentially the same line of reasoning.

Adams (1972) explained this result heuristically by noting another aspect of the simulations, namely that photons escape the medium only in the far line wings of the Voigt profile $\phi(x)$, where $x$ is a normalized frequency displacement from line center defined just 
below. They escape at $\left|x_{*}\right| \equiv\left|\left(\nu_{*}-\nu_{0}\right)\right| / \Delta \nu_{\mathrm{D}} \approx\left(a \tau_{0}\right)^{1 / 3}$ where $\Delta \nu_{\mathrm{D}}=\left(\nu_{0} / c\right) \sqrt{2 \ln (2) k T_{\mathrm{D}} / m_{\mathrm{H}}}$ is the Doppler $H W H M$ and a is the damping constant measured in units of $\Delta \nu_{\mathrm{D}}$. Although most scattering occurs nearer the Doppler core, photons move appreciably in space only when they scatter at frequencies where the medium is less opaque. The photons escape at a frequency where the optical depth is still well above unity; $\left|x_{*}\right| /\left|x_{\tau=1}\right|=$ $0.285\left(T_{\mathrm{D}} / 10^{4} \mathrm{~K}\right)^{1 / 6}\left(N_{\mathrm{H}} / 10^{20} \mathrm{~cm}^{-2}\right)^{-1 / 6}$ where $\pm x_{\tau=1}$ are the frequency shifts at which the optical depth drops to unity for a pure damping profile.

Given that the rms Dopper shift in any scattering is $\left|\Delta \nu_{\mathrm{D}}\right|$ (the mean shift is 0 in the core and $-1 /|x|$ in the far wings), Adams reasoned that, occasionally, a photon would experience one series of scatterings into the far wings carrying it at once $\left|x_{*}\right|$ frequency units from the line center and entirely out of the scattering medium. If a photon must travel a distance $\tau_{0}$ to escape, $x_{*}$ should satisfy the condition $x_{*} / \phi\left(x_{*}\right)=\tau_{0}$ since the scattering length is proportional to $1 / \phi(x)$. This recovers the behaviour seen in the simulations since $\phi(x) \propto a / x^{2}$ in the damping wings.

Shortly after this work, the transfer equation was effectively solved in closed form by Harrington (1973) in the case of very large optical depths $\left(a \tau_{0}\right)^{1 / 3}>1$. The behaviour seen in the simulations of Adams (1972) was recovered analytically in the change of variables needed to effect the solution: to something which varies as $x^{3}$ in the line wings. The numerical work was extended by Adams (1975) and Hummer \& Kunasz (1980), and the analytic work by Neufeld $(1990,1991)$. There, it was shown that the mean distance travelled by a Ly- $\alpha$ photon is actually $\left(a \tau_{0}\right)^{1 / 3} \tau_{0}$. Because $a=0.0425 / T_{\mathrm{D}}{ }^{0.5}$ is typically a small number, the cube root factor is of order $10-100$. This description is correct to factors of order unity, as discussed by Hummer \& Kunasz (1980).

For reference, we note that the line center optical depth $\tau_{0}=5.9010^{-12} N_{\mathrm{H}} / T_{\mathrm{D}}^{0.5}$ is proportional to $A_{\alpha} / \Delta \nu_{\mathrm{D}}$, which is the damping constant $a$ except for a numerical factor. Thus $a \tau_{0}=1.3910^{-10} a^{2} N_{\mathrm{H}}=2.5110^{-13} N_{\mathrm{H}} / T_{\mathrm{D}}$. If $T_{\mathrm{D}} \approx T_{\mathrm{K}}$, the latter ratio of column density to temperature is familiar from the expression for the integrated optical depth of the hyperfine line. Thus we come full circle and find (but do not intend to claim a causal connection!) that the crucial parameter for Ly- $\alpha$ excitation of the $\lambda 21 \mathrm{~cm}$ line is proportional to the $\lambda 21 \mathrm{~cm}$ optical depth ${ }^{2}$.

\subsection{Internal sources of $L y-\alpha$ photons}

We included three locally generated sources of Ly- $\alpha$ photons in H I gas: electron impact excitation of the ${ }^{2} \mathrm{P}$ level using the rate constant calculated by Scholz \& Walters (1991); electron-proton recombinations into levels $n \geq 2$ (except ${ }^{2} \mathrm{~S}$ ) for which the relevant coefficients are summarized in Table 1 and for which our rates are very slight

\footnotetext{
2 The discussion of the previous two paragraphs neglects internal absorption, which is discussed in detail in Sect. 5.3.
}

upper limits; and excitation by the same cosmic- and X-ray fluxes which ionize the gas. For the latter, we follow Draine \& Salpeter (1978) and assume that 1.5 Ly- $\alpha$ photons are generated per primary ionization event.

In general, electron-impact excitation is unimportant except above $10^{4} \mathrm{~K}$ and cosmic-ray and X-ray generated photons dominate in the denser gas.

\subsection{The locally-generated $L y-\alpha$ photon field in a dusty H I cloud}

The volume rate at which Ly- $\alpha$ photons are created, $\dot{n}_{\alpha}$, is straightforward to specify. But for the excitation rate we require in Field's formulation the volume density of photons $n_{\alpha}=\dot{n}_{\alpha} \times t$, where $t$ is a characteristic retention time (the expression could just as well have been cast in terms of the local mean intensity). In fact, following Field (1958), the relevant time is $R / c$ where $2 R$ is the characteristic size of the medium. Although this seems naive, it is supported by the analytic solutions of Harrington (1973) which we now discuss: the actual distance travelled by a photon, $\left(a \tau_{0}\right)^{1 / 3} \tau_{0}$, is associated with a broadening of the profile by an amount $\left(a \tau_{0}\right)^{1 / 3}$, leaving the line-center Ly- $\alpha$ intensity unchanged.

Harrington (1973) presents an analytic solution for the case of a uniform slab with a uniform internal source of Ly- $\alpha$ radiation $\dot{n}_{\alpha}$ such that 1 photon is created per unit area per unit time looking down through a slab of half-thickness $R$. To first approximation, the mean intensity at the slab midplane, assuming a lossless medium, is $J(\tau=0, x)=\left(\sqrt{6} / 2 \pi^{3}\right) \exp \left(-1.34|x|^{3} /\left(a \tau_{0}\right)\right)$. To convert this normalized solution to "real" units, the density of photons at the line center is just $4 \pi / c \times 2 R \times J(0,0)=$ $\left(4 \sqrt{6} / \pi^{2}\right) \times(R / c)=0.993 R / c \equiv f \times R / c$.

A slightly more accurate expression for the photon field is given by Harrington (1973) as a series which includes the possibility of accounting for losses by continuous absorption in the medium. To calculate the loss it is necessary to evaluate the term $(\sqrt{6} / 2) \epsilon \tau_{0}$ where $\epsilon$ is the (assumed small) probability of absorption per scattering (recall that there are on average $\tau_{0}$ scatterings). The mean distance travelled per scattering, measured in units of the distance necessary to traverse one optical depth at the line center, is $\left(a \tau_{0}\right)^{1 / 3}$ (Adams 1975; Neufeld 1990); the physical distance corresponding to one optical depth at the line center is found by equating $\Delta \tau_{0}=5.9010^{-12} n_{\mathrm{H}} \times \Delta l / \sqrt{T_{\mathrm{D}}}=1$. From this it follows that $\epsilon \tau_{0}=\left(a \tau_{0}\right)^{1 / 3} N_{\mathrm{H}} \sigma_{\mathrm{gr}}$ abs where $\sigma_{\mathrm{gr}}{ }^{\text {abs }}=1.2710^{-21} \mathrm{~cm}^{-2}$ has been calculated recently by Weingartner \& Draine (2001) (their value for the albedo is 0.32 ). Several cases of lossy media are treated at length by Neufeld (1990) (analytically) and by Adams (1971a) (numerically). The formalism discussed here reproduces the numerical results in the latter reference quite well but the grain model of Weingartner \& Draine (2001) is about $30 \%$ more absorptive than the worst case considered by Adams. 


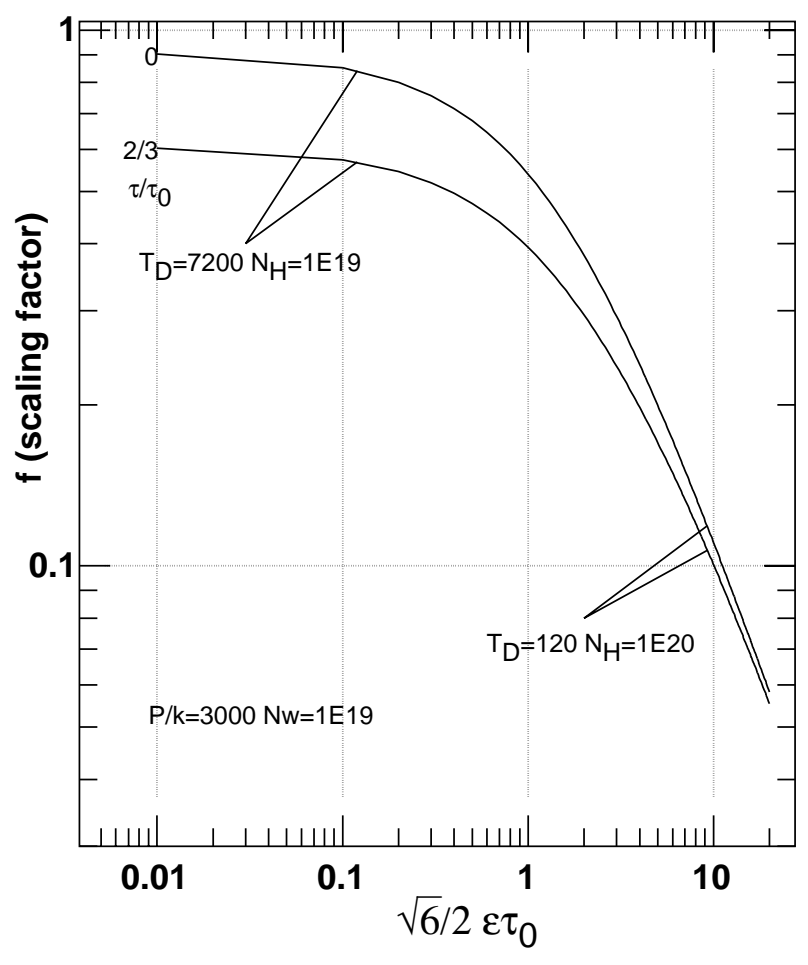

Fig. 4. A scaling factor for the mean intensity inside a lossy slab harboring a uniform source of Ly- $\alpha$ radiation, following Eqs. (27) and (41) of Harrington (1973); see Sect. 5.3 here. Values are shown at the midplane $\left(\tau / \tau_{0}=0\right)$ and two-thirds of the way from the midplane to the slab surface. Relevant values for warm and cool neutral gas are indicated. These calculations are the origin of the correction factor displayed in the prior figure

In Fig. 4 we show the scale factor $f$ multiplying $R / c$ as a function of $\epsilon \tau_{0}$. For a lossless medium, the exact analytic solution yields $f=0.909$ at the line center and slab midplane (vs. 0.993). Losses are relatively unimportant in the intercloud gas over columns which are only a few pc long, i.e. those which might be associated with individual cloud envelopes (the case where the size of the medium is comparable to the galactic scale height is considered in the next section). But the attenuation of $\mathrm{Ly}-\alpha$ radiation will be significant in any cool gas which absorbs strongly at $\lambda 21 \mathrm{~cm}$.

\subsection{Efficacy of the locally-generated $L y$ - $\alpha$ photon field}

Figure 3 is a behind-the-scenes look at the excitation of the hyperfine line as it occurs under the conditions used to produce Figs. 1, 2; it shows the $y$-values for collisions and Ly- $\alpha$ excitation. For the purposes of the calculation, we took a characteristic size $R=1.5$ pc. Ly- $\alpha$ excitation would compete strongly with particle excitation in cooler gas were it not for the effects of dust absorption and the decrease in ionization fraction due to recombination on grains. In warmer gas, the small physical size of the medium assumed for the calculation limits the effectiveness of Ly- $\alpha$ excitation except where the gas ionizes fully.
We conclude that there is no locally-generated source of excitation within individual clouds which will suffice to thermalize the spin temperature of warm $\mathrm{H}$ I, although the small attenuation in warm gas means that Ly- $\alpha$ excitation can become important if galaxian scales are considered. Because the Galaxy is a copious producer of Ly- $\alpha$ photons, the galactic Ly- $\alpha$ radiation field will dominate excitation of the $\lambda 21 \mathrm{~cm}$ line under some assumed conditions, as discussed below.

\section{Ly- $\alpha$ excitation by Galactic radiation}

The space-averaged volume creation rate of Ly- $\alpha$ photons near the galactic plane dwarfs that generated in situ in $\mathrm{H} \mathrm{I}$ gas by large factors. The galactic density of Ly- $\alpha$ photons is not known with certainty but estimates are enough to force consideration of its importance in exciting the neutral H I in warm media.

Vacca et al. (1996) have shown that the areal production rate of ionizing photons from 429 O- and early-B stars within $2.5 \mathrm{kpc}$ of the Sun is $\Psi_{0}=3.710^{7}$ photons $\mathrm{cm}^{-2} \mathrm{~s}^{-1}$. Each ionizing photon should produce of order one Ly- $\alpha$ photon. Thus, over a cylinder of height $h= \pm 50 \mathrm{pc}=100 \mathrm{pc}$ about the Galactic midplane, the mean volume production rate is $\dot{n}_{\alpha} \approx \Psi_{0} / h=$ $1.210^{-13} \mathrm{~cm}^{-3} \mathrm{~s}^{-1}$. This is far higher than that generated solely by recombination in the warm neutral gas we modelled: the combination of a long path and higher volume production rate would result in a vastly increased line excitation by Ly- $\alpha$ photons ${ }^{3}$.

In Fig. 5 we show the behaviour of $T_{\mathrm{sp}}$ when a specified fraction $F_{\alpha}$ of the galactic Ly- $\alpha$ flux threads the gas assuming $N w=10^{19} \mathrm{~cm}^{-2}$ and $T_{\mathrm{L}}=T_{\mathrm{K}}$. Behaviour in the upper left panel of Fig. 5 is identical to that in Fig. 2. Figure 5 shows that even a very small fraction of the galactic Ly- $\alpha$ radiation suffices to dominate the excitation.

Apparently, there is an abundant source of noncollisional excitation, if only it can be used.

\subsection{A two-phase ISM}

We consider first an idealized two-phase interstellar medium which fills all space, and in which nearly all of the volume is occupied by warm intercloud gas. Following the discussion of this problem by Adams (1971a) we idealize the thin layer of young stars to be a plane source embedded in the much thicker, warm H I layer, and apply an analytic radiative transfer solution using the new value of the grain absorption cross-section from Weingartner \& Draine (2001) as mentioned earlier. At first, we neglect

${ }^{3}$ This value of $\Psi_{0}$ is three times higher than that of Draine \& Salpeter (1978) who added the separate contributions of H II regions, recombination in diffuse hydrogen, and production in supernova remants (any such extra contributions should in fact be included). Adams (1971b) cites an observational limit on the galactic Ly- $\alpha$ flux due to Thomas \& Krassa (1971) but this limit is consistent with large values of $\Psi_{0}$ given recent estimates of the continuous absorption by dust. 

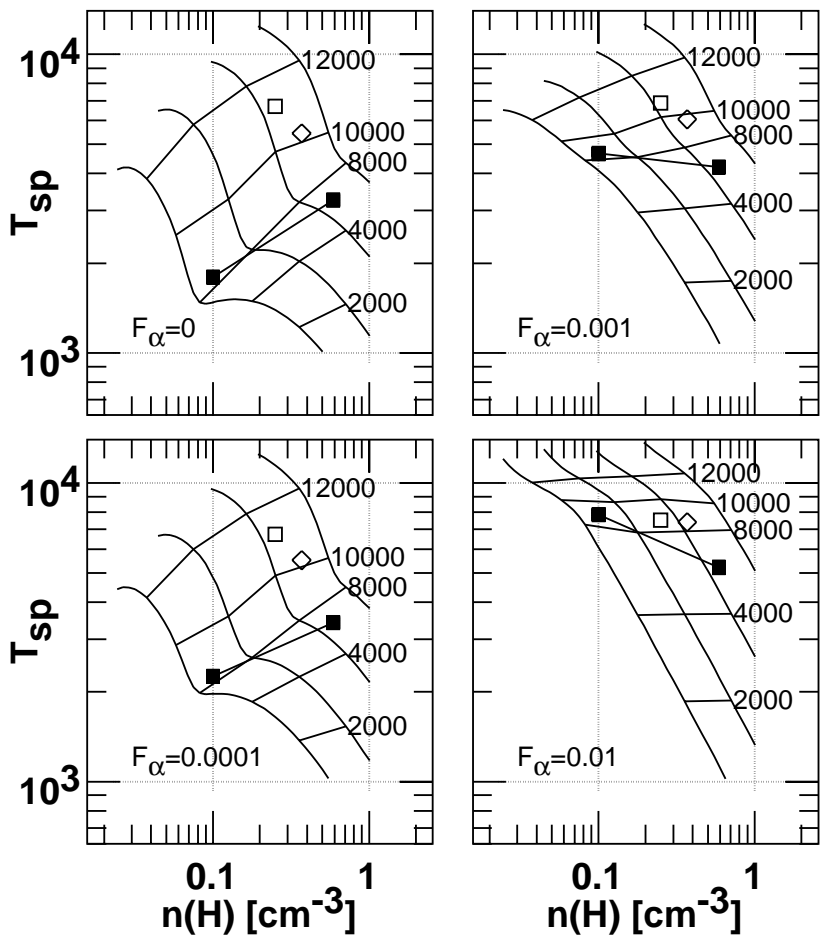

Fig. 5. As in Fig. 2 when a specified fraction $F_{\alpha}$ of the galactic flux from early-type stars threads the gas and $T_{\mathrm{L}}=T_{\mathrm{K}}$. The panel with $F_{\alpha}=0$ repeats behaviour already shown in Fig. 2

the fact that most photons which enter cold H I clouds will be absorbed by dust.

Figure 6 shows our evaluation of the analytic solution (Harrington 1973; Neufeld 1990) to this idealized problem, performed for a gas with $T_{\mathrm{K}}=T_{\mathrm{D}}=7200 \mathrm{~K}$ and various values of the column density in the $\mathrm{H}$ I layer (which determines the loss due to continuous absorption by dust). Although the total column density of $\mathrm{H}$ I looking out from the midplane is about $310^{20} \mathrm{~cm}^{-2}$ (Liszt 1983), that of the intercloud gas is somewhat less, of order half. Figure 6 shows that over most of the volume of this idealized model, the Ly- $\alpha$ radiation field threading the intercloud gas is certainly not less than $1 \%$ of $\Psi_{0}$. In this case, the spin temperature in the intercloud gas at all but the highest galactic $z$-heights would be close to equilibrium with the temperature of the ambient galactic Ly- $\alpha$ radiation $-T_{\mathrm{K}}$, $T_{\mathrm{D}}$ or what have you, depending on how the photon field relaxes in its interactions with the gas.

Cool neutral gas packets dispersed throughout such a scattering medium would clearly be threaded by a substantial fraction of the galactic flux as well, raising the interesting possibility that the spin temperatures measured in strongly absorbing gas are also influenced by Ly- $\alpha$ radiation. A calculation analogous to that used to produce Fig. 5 shows that Ly- $\alpha$ excitation dominates if more than $0.1 \%$ of the galactic radiation field threads cool gas. In this case, $T_{\mathrm{sp}}=T_{\mathrm{D}}$ rather than $T_{\mathrm{sp}}=T_{\mathrm{K}}$ and typical turbulent velocity contributions to $T_{\mathrm{D}}$ will put a $30-120 \mathrm{~K}$ floor on measurements of $T_{\mathrm{sp}}$ (see Sect. 6.3) if the turbulence is established on the scale of the Ly- $\alpha$ scattering.

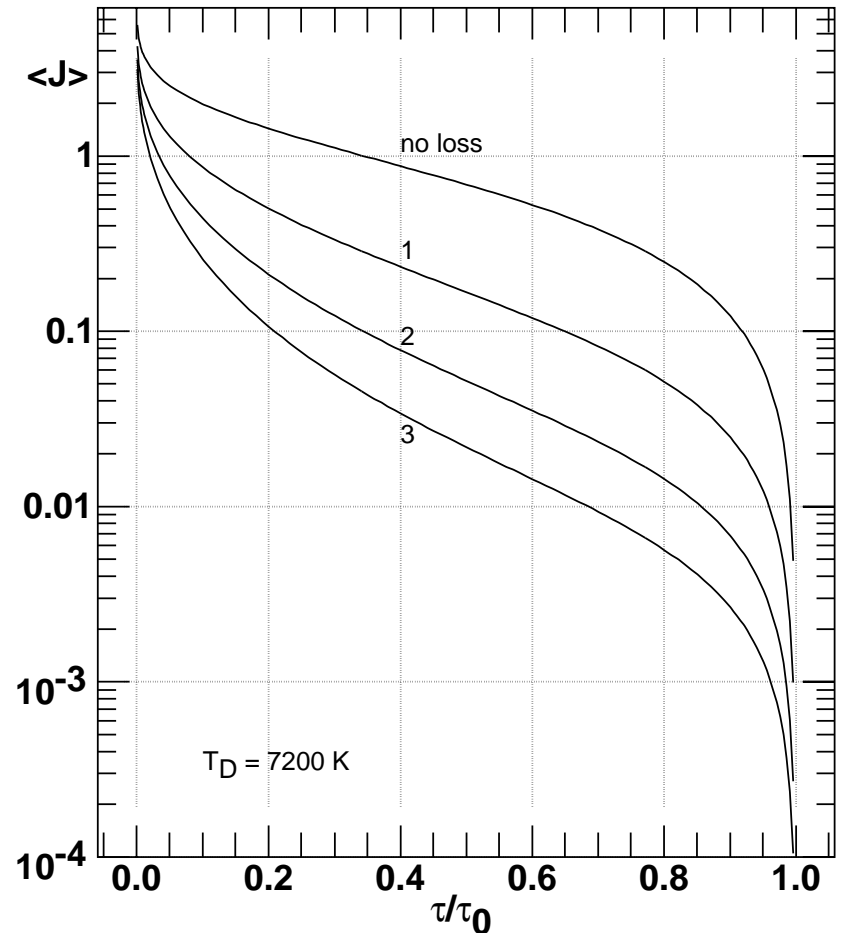

Fig. 6. Variation of the Ly- $\alpha$ radiation field inside a slab of warm $\mathrm{H}$ I harboring a unit plane source $\delta(\tau)$ at the midplane, plotted against distance from the slab center $\tau / \tau_{0}$; see Sect. 6.1 of the text. Results for a lossless slab are shown, and for $N_{\mathrm{H}}=$ 1,2 , and $310^{20} \mathrm{~cm}^{-2}$

\subsection{A multi-phase ISM}

Neufeld (1991) pointed out that a multiphase ISM structure may foster escape of Ly- $\alpha$ radiation and that individual neutral gas clouds - warm or cool - may not be threaded by much of the ambient Ly- $\alpha$ flux. If most of the volume of the ISM is in a contiguous hot phase having negligible neutral hydrogen or Ly- $\alpha$ absorption, scattered radiation escapes relatively easily even when neutral gas clouds have a very high surface covering factor. Photons scatter off individual clouds as if they were particles of a gas having a Doppler temperature corresponding to the cloud-cloud velocity dispersion, $6 \mathrm{~km} \mathrm{~s}^{-1}$, appreciably narrowing the interstellar line profile if the photons are scattered sufficiently often before escape. The crucial issue is the fraction and connectedness of the ISM occupied by very hot gas in which hydrogen is totally ionized, as opposed even to $6000-10000 \mathrm{~K} \mathrm{H}$ I intercloud gas which will still present a sizable opacity to Ly- $\alpha$ radiation.

The fraction of incident photons which will be reflected back from the outer boundary of an isolated, discrete cloud is of order $1-1 /\left(\tau_{0} \phi(x)\right)$ where $x$ is the normalized frequency shift of the incident radiation from line center (Neufeld 1990): see Sect. 5.1 here. An analytic solution to the problem of radiation incident on a lossless slab is presented by Neufeld (1990) in his Eqs. (2.19)-(2.22); calculation is considerably simplified when Neufeld's Eq. $(2.22)^{4}$

\footnotetext{
${ }^{4}$ Note that the quantity plotted in Fig. 2 of Neufeld (1990) is $\left(a \tau_{0}\right)^{1 / 3} J\left(\tau_{0}, x\right)$, not $\left(a \tau_{0}\right) J\left(\tau_{0}, x\right)$.
} 


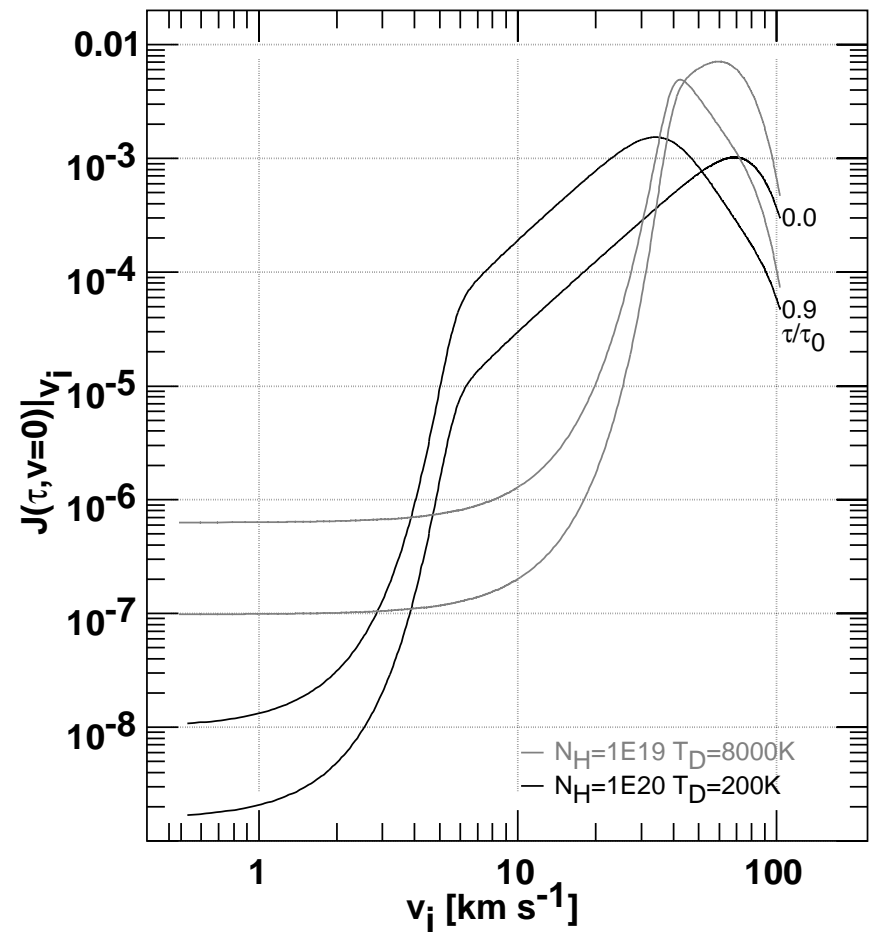

Fig. 7. Line-center Ly- $\alpha$ mean intensity inside a lossless slab in the presence of a half-unit monochromatic flux $\delta\left(v-v_{i}\right) / 2$ incident on each face following the analytic solution of Neufeld (1990). Two combinations of slab thickness and Doppler temperature are shown, representing cool and warm neutral gas. For each of these, the mean intensity is shown at the center and $90 \%$ of the way from the mid-plane to the slab surface. Over most of the interior, the behaviour more closely resembles that at the midplane

is recast as

$$
\begin{aligned}
F(w, y) & =g \times \ln (1-2 \exp (-\pi y) \cos (\pi w)+\exp (-2 \pi y)), \\
g & \equiv \sqrt{6} /\left(16 \pi^{2}\right) .
\end{aligned}
$$

In Fig. 7 we show the mean intensity at the line center inside lossless slabs of warm and cool gas as the frequency shift of the attempted injection (expressed in $\mathrm{kms}^{-1}$ ) varies; neglect of loss is appropriate for warm gas on the scale of individual clouds. Any cool gas in the ISM is either embedded in a strongly-scattering intercloud gas or enrobed in warmer $\mathrm{H}$ I and its internal radiation field could be much larger than that shown here; the reflectivity calculations for bare cool gas are included only to illustrate parameter sensitivities.

If the ambient Ly- $\alpha$ photon field is as narrow as the $6 \mathrm{~km} \mathrm{~s}^{-1}$ cloud-cloud velocity dispersion after multiple reflections, little will penetrate cool gas and essentially none will enter the warm H I. But if the Ly- $\alpha$ photon field is appreciable at relative velocities of $30-100 \mathrm{~km} \mathrm{~s}^{-1}$ with respect to the line center, the internal radiation field in warm neutral gas could be as high as $0.1-1 \%$ of that which is incident. The interstellar Ly- $\alpha$ field would be quite broad in any model where the galactic layer harbors appreciable amounts of distributed warm H I (Adams 1971b) and Ly- $\alpha$ photons will be injected into the ISM in the local line

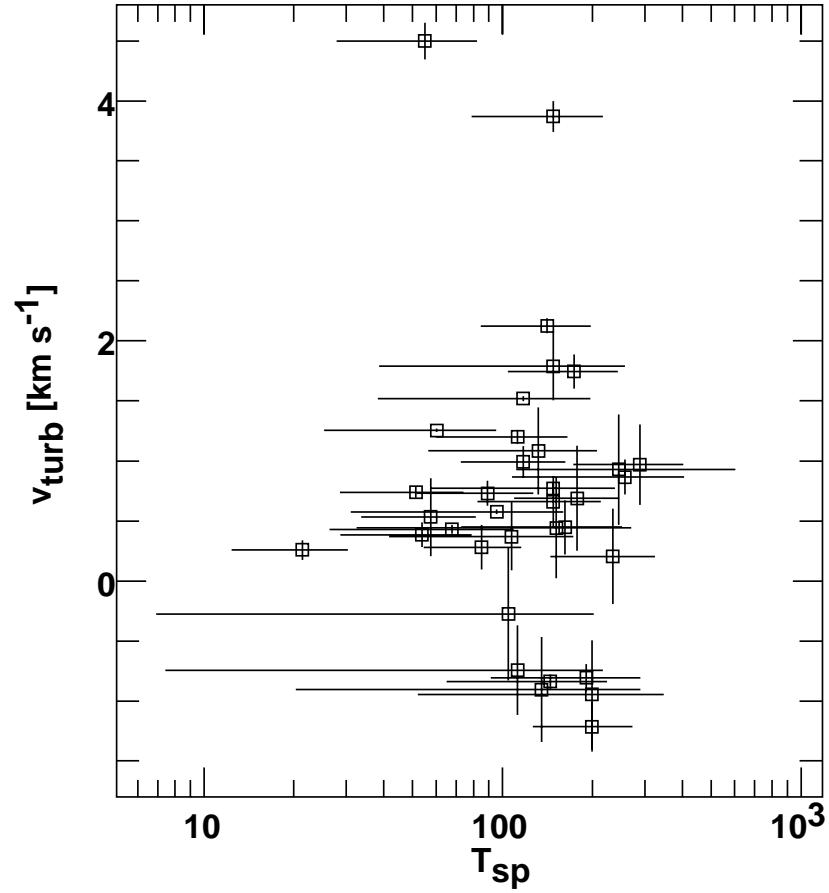

Fig. 8. Turbulent velocity contributions derived from the spin and Doppler-temperature measurements of Payne et al. (1982, $1983)$ using $\left(T_{\mathrm{D}}=T_{\mathrm{sp}}+121 v_{\mathrm{turb}}{ }^{2}\right)$ for features having $\tau_{0} \geq$ 0.04

wings when they escape an $\mathrm{H}$ II region. But the ambient Ly- $\alpha$ radiation field and its Doppler temperature are not easily specified in extreme three-phase models in which no Ly- $\alpha$ scattering occurs between individual clouds (Neufeld 1991).

\subsection{Non-thermal Doppler excitation}

For cool gas, where Ly- $\alpha$ excitation would be important if galactic radiation enters via the intercloud or warm neutral gas which enrobes it, HI absorption features typically show a noticeable difference between $T_{\mathrm{sp}}$ and $T_{\mathrm{D}}$, implying that excitation of the hyperfine transition is not dominated by Ly- $\alpha$ excitation at a temperature other than $T_{\mathrm{K}}$.

In Fig. 8, we show the turbulent velocity contributions derived from the measurements of Payne et al. (1982, 1983) where, for the stronger features $(\tau \geq 0.04)$ and excepting a few cases with very large quoted errors in $T_{\mathrm{sp}}$, we have derived values of $v_{\text {turb }}$ according to Eq. (3). In those few cases where $T_{\mathrm{D}}<T_{\mathrm{sp}}$ in the data, the value is shown negative. The error estimates are ours, derived from analysis of the errors quoted in the original data.

Clearly, most of the data are consistent with an added microturbulent contribution of order $v_{\text {turb }}=$ $0.5-1.0 \mathrm{~km} \mathrm{~s}^{-1}$, equivalent to adding a $30-120 \mathrm{~K}$ turbulent contribution in quadrature with $T_{\mathrm{K}}$ to form $T_{\mathrm{D}}$. Of course, if Ly- $\alpha$ excitation is actually operating at a Doppler temperature above $T_{\mathrm{K}}$, the measured spin temperatures are already somewhat inflated, and the turbulent velocities 
extracted from the comparison between $T_{\mathrm{sp}}$ and $T_{\mathrm{D}}$ are only lower limits. Statistically, $\left\langle T_{\mathrm{sp}} / T_{\mathrm{D}}\right\rangle=0.56 \pm 0.26$ in this dataset.

Recent studies of stray-radiation-corrected Galactic emission profiles find evidence for three kinematic components having linewidths of 5,13 , and $31 \mathrm{~km} \mathrm{~s}^{-1}$ (Verschuur $\&$ Peratt 1999). These correspond respectively to individual clouds (or to $T_{\mathrm{D}}=550 \mathrm{~K}$; see Takakubo 1967, and references therein), to the $6 \mathrm{~km} \mathrm{~s}^{-1}$ cloud-cloud velocity dispersion (or $T_{\mathrm{D}}=3700 \mathrm{~K}$ ), and to an anonymous component with $T_{\mathrm{D}}=21000 \mathrm{~K}$ or a combination of $T_{\mathrm{K}}=8000 \mathrm{~K}, v_{\text {turb }}=10.4 \mathrm{~km} \mathrm{~s}^{-1}$. Suggestions of a pure intercloud component corresponding to $T_{\mathrm{K}}=8000 \mathrm{~K}$, $v_{\text {turb }}=0 \mathrm{~km} \mathrm{~s}^{-1}$ have a somewhat checkered history, but seem now not to be clearly evident in the data. This is perhaps not too surprising given the panoply of phenomena which may influence an observed linewidth on galactic scales.

\section{Summary}

The major conclusions of this work may be summarized as follows:

Particle excitation does not thermalize warm (intercloud) H I in either the two- or three-phase models of the ISM. In the absence of other sources of excitation, one would expect to see $T_{\mathrm{sp}} \approx 1000-5000 \mathrm{~K}$, from regions where $T_{\mathrm{K}} \approx 5000-10000 \mathrm{~K}$ (note that regions of higher $T_{\mathrm{K}}$ typically are at lower pressure in two-phase equilibrium and produce lower $T_{\mathrm{sp}}$ ), even when the contribution of warm gas can be isolated. The presence of a wide range of $T_{\mathrm{sp}}$ in warm gas complicates the derivation of $T_{\mathrm{sp}}$ in cool gas, which is nearly always seen blended with warm gas along the line of sight.

In the two-phase model, at all but the highest galactic $z$-heights, hyperfine excitation in warm, neutral intercloud gas will generally be dominated by the scattering of galactic Ly- $\alpha$ photons produced by the ensemble of OB stars and supernova remnants, in which case the spin temperature will correspond to whatever Doppler temperature the Ly- $\alpha$ photon field acquires while interacting with the interstellar gas. Alternatively - recalling comments in the Introduction - excitation may be dominated locally by the Ly- $\alpha$ radiation originating in or near particular objects.

In the three-phase model the ability of the galactic Ly- $\alpha$ radiation field to penetrate individual $\mathrm{H}$ I clouds is somewhat problematic.

In any multi-phase model where the warm neutral gas is threaded by a substantial galactic Ly- $\alpha$ flux, excitation in the cool $\mathrm{H}$ I will be influenced by the Ly- $\alpha$ photon field also.

The excitation in cool H I clouds seems not to be totally dominated by Ly- $\alpha$ excitation at a temperature other than $T_{\mathrm{K}}$, judging from the ubiquity of an apparent turbulent velocity contribution to the $\mathrm{H}$ I absorption linewidth, i.e. $\left\langle T_{\mathrm{sp}} / T_{\mathrm{D}}\right\rangle \approx 0.56$.

The scattering calculations which will tell us the color temperature of the interstellar Ly- $\alpha$ radiation field remain to be performed and may require more knowledge of the structure of the ISM than is currently available to us.

Acknowledgements. The National Radio Astronomy Observatory is operated by AUI, Inc. under a cooperative agreement with the US National Science Foundation. I thank David Neufeld and John Dickey for helpful comments on the manuscript and Fred Schwab for providing a reference to a really good numerical representation of the Voigt function.

\section{References}

Adams, T. F. 1971a, ApJ, 168, 575

Adams, T. F. 1971b, A\&A, 12, 280

Adams, T. F. 1972, ApJ, 174, 439

Adams, T. F. 1975, ApJ, 201, 350

Allison, A. C., \& Dalgarno, A. 1969, ApJ, 158, 423

Bahcall, J. N., \& Ekers, R. D. 1969, ApJ, 157, 1055

Balucinska-Church, M., \& McCammon, D. 1992, ApJ, 400, 699

Black, J. H., \& Dalgarno, A. 1977, ApJS, 34, 405

Braskén, M., \& Kyrölä, E. 1998, A\&A, 332, 732

Davies, R. D., \& Cummings, E. R. 1975, MNRAS, 170, 95

Dickey, J. M., Terzian, Y., \& Salpeter, E. E. 1978, ApJS, 36, 77

Draine, B. T., \& Salpeter, E. E. 1978, Nature, 271, 730

Draine, B. T., \& Sutin, B. 1987, ApJ, 320, 803

Field, G. B. 1958, IRE, 57, 31

Field, G. B. 1959, ApJ, 129, 551

Harrington, J. P. 1973, MNRAS, 162, 43

Hummer, D. G., \& Kunasz, P. B. 1980, ApJ, 236, 609

Leung, C. M., \& Liszt, H. S. 1976, ApJ, 208, 732

Liszt, H. S. 1983, ApJ, 275, 163

Martin, P. G. 1988, ApJS, 66, 125

McKee, C. F., \& Ostriker, J. P. 1977, ApJ, 218, 148

Neufeld, D. A. 1990, ApJ, 350, 216

Neufeld, D. A. 1991, ApJ, 370, L85

Payne, H. E., Salpeter, E. E., \& Terzian, Y. 1983, ApJ, 272, 540

Payne, H. E., Terzian, Y., \& Salpeter, E. E. 1982, ApJS, 48, 199

Scholz, T. T., \& Walters, H. R. J. 1991, ApJ, 380, 302

Smith, F. J. 1966, Planet. Space Sci., 14, 929

Takakubo, K. 1967, B. A. N., 19, 125

Thomas, G. E., \& Krassa, R. F. 1971, A\&A, 11, 218

Urbaniak, J. J., \& Wolfe, A. M. 1981, ApJ, 244, 406

Vacca, W. D., Garmany, C. D., \& Shull, J. M. 1996, ApJ, 460, 914

Verschuur, G. L., \& Peratt, A. L. 1999, AJ, 118, 1252

Weingartner, J. C., \& Draine, B. T. 2001, ApJ, 548, in press

Wolfire, M. G., Hollenbach, D., McKee, C. F., Tielens,

A. G. G. M., \& Bakes, E. L. O. 1995, ApJ, 443, 152

Wouthuysen, S. A. 1952, AJ, 57, 31

Zanstra, H. 1949, B. A. N., 11, 1 